\title{
Optically and microwave induced magnetization precession in [Co/Pt]/NiFe exchange springs
}

Maciej Dabrowski, ${ }^{*, \dagger}$ Andreas Frisk, ${ }^{\ddagger}$ David M. Burn, ${ }^{\ddagger}$ David G. Newman, ${ }^{\dagger}$

Christoph Klewe, ${ }^{\text {II }}$ Alpha T. N'Diaye, ${ }^{\text {II }}$ Padraic Shafer, ${ }^{\text {II }}$ Elke Arenholz, II,

Graham J. Bowden, " Thorsten Hesjedal, ${ }^{\perp}$ Gerrit van der Laan, ${ }^{\ddagger}$ Gino Hrkac, ${ }^{\#}$ and Robert J. Hicken ${ }^{\dagger}$

tDepartment of Physics and Astronomy, University of Exeter, Stocker Road, Exeter, Devon EX4 4QL, United Kingdom

$\ddagger$ Diamond Light Source, Harwell Science and Innovation Campus, Didcot, OX11 oDE, United Kingdom

IIAdvanced Light Source, Lawrence Berkeley National Laboratory, Berkeley, California 94720, USA

§Cornell High Energy Synchrotron Source, Cornell University, Ithaca, New York 14853, USA \|School of Physics and Astronomy, University of Southampton, SO17 1BJ, United Kingdom $\perp$ Department of Physics, Clarendon Laboratory, University of Oxford, OX1 $3 P U$, United Kingdom

\#College of Engineering, Mathematics and Physical Sciences, University of Exeter, Stocker Road, Exeter, Devon EX4 4QL, United Kingdom

E-mail: m.k.dabrowski@exeter.ac.uk 


\begin{abstract}
Microwave and heat assisted magnetic recording are two competing technologies that have greatly increased the capacity of hard disk drives. The efficiency of the magnetic recording process can be further improved by employing non-collinear spin structures that combine perpendicular and in-plane magnetic anisotropy. Here, we investigate both microwave and optically excited magnetization dynamics in $[\mathrm{Co} / \mathrm{Pt}] / \mathrm{NiFe}$ exchange spring samples. The resulting canted magnetization within the nanoscale $[\mathrm{Co} / \mathrm{Pt}] / \mathrm{NiFe}$ interfacial region allows for optically stimulated magnetization precession to be observed for an extended magnetic field and frequency range. The results can be explained by formation of an imprinted domain structure, which locks the magnetization orientation and makes the structures more robust against external perturbations. Tuning the canted interfacial domain structure may provide greater control of optically excited magnetization reversal and optically generated spin currents, which are of paramount importance for future ultrafast magnetic recording and spintronic applications.
\end{abstract}

\title{
Keywords
}

interfacial domains, exchange spring magnets, ferromagnetic resonance, time resolved magneto-optical Kerr effect (TRMOKE), x-ray magnetic circular dichroism (XMCD), xray detected ferromagnetic resonance (XFMR), spin transfer torque (STT) 


\section{INTRODUCTION}

Magnetic materials play an essential role in the efficient utilization of sustainable energy resources. ${ }^{1}$ Exchange spring (ES) magnets are formed from exchange coupled hard and soft magnetic layers and have been explored in the context of high-performance permanent magnets. ${ }^{2}$ In recent years, research into ES magnets has received fresh impetus due to the promise of microwave assisted ${ }^{3}$ and optically induced ${ }^{4}$ magnetization switching, which are the fundamental processes exploited within microwave-assisted magnetic recording $(\mathrm{MAMR})^{5}$ and heat-assisted magnetic recording (HAMR). ${ }^{6}$ While different ES structures with in-plane magnetic anisotropy have been extensively studied over the past three decades, ${ }^{2,7}$ the so called out-of-plane ES magnets, compromised of layers with out-of-plane and in-plane magnetic anisotropy, are still relatively unexplored. ${ }^{8,9}$ The combination of layers with in-plane and out-of-plane magnetic anisotropies allows a non-collinear spin structure to be created in which the tilting of the magnetization can be tuned. ${ }^{10,11}$ Such structures are of great technological interest since they can also improve the efficiency and versatility of spin transfer torque (STT $)^{12-14}$ and spin orbit torque $(\mathrm{SOT})^{15}$ devices.

While microwave field driven STT and spin current phenomena have been thoroughly studied ${ }^{16-22}$ there is a growing interest in ultrafast transfer of spin angular momentum mediated by optically induced hot electron spin current on sub-picosecond timescales. ${ }^{23-25}$ To observe optically induced STT, an additional bias magnetic field is usually needed to break the symmetry and provide an initial torque on the magnetization of the sink layer. ${ }^{23,26}$ Out-of-plane ES magnets should be ideal candidates for such studies since their tilted magnetization allows the absorbed spin current to exert greater torque.

The most well explored out-of-plane ES structures are composed of two sub-units, one with perpendicular anisotropy such as multilayered $[\mathrm{Co} / \mathrm{Pd}]^{10,11,27,28}$ or a $\mathrm{L}_{1}-\mathrm{CoPt}$ layer, ${ }^{29-32}$ and the other with a small in-plane anisotropy (usually NiFe). However, stud- 
ies have been mostly limited to static magnetic properties so far, and further experiments providing insight into the magnetization dynamics are highly desirable.

In this work, we focus on the dynamic response to both microwave and optical excitation and study the effect of canted magnetization in $[\mathrm{Co}(0.4) / \mathrm{Pt}(0.9)]_{n} / \mathrm{Ni}_{81} \mathrm{Fe}_{19}($ d) hybrid structures, where $n$ indicates the number of $\mathrm{Co} / \mathrm{Pt}$ repeats and $d$ is the $\mathrm{Ni}_{81} \mathrm{Fe}_{19}$ layer thickness (all layer thicknesses in parentheses given in $\mathrm{nm}$ and the sub-units are referred to as $[\mathrm{Co} / \mathrm{Pt}]$ and $\mathrm{NiFe}$ from hereon). We choose $[\mathrm{Co} / \mathrm{Pt}]$ multilayers as they are currently of interest for all-optical switching (AOS), ${ }^{33}$ with the switching mechanism being the subject of continuing debate. ${ }^{34-36}$ We demonstrate that the magnetization precession within $[\mathrm{Co} / \mathrm{Pt}]_{n} / \mathrm{NiFe}$ is strongly dependent upon the type of excitation. For microwave driven ferromagnetic resonance (FMR), heavily damped magnetization precession is observed for magnetic fields smaller than the saturation field. We directly measure the magnetization precession of the individual layers by using element specific x-ray detected ferromagnetic resonance $(\mathrm{XFMR})^{37-40}$ and show that tilted magnetization within the $[\mathrm{Co} / \mathrm{Pt}]$ sub-unit results in a precession cone angle that is larger than that for the NiFe layer. In contrast, upon excitation with ultrashort laser pulses, the magnetization precession can be observed over an increased frequency range of 2.5 to $21 \mathrm{GHz}$, and up to the highest available magnetic induction of $1.5 \mathrm{~T}$. This suggests a mutual imprinting of a non-uniform magnetic configuration between the $[\mathrm{Co} / \mathrm{Pt}]$ and $\mathrm{NiFe}$ sub-units that is mediated by the interfacial exchange interaction. ${ }^{41-43}$

\section{RESULTS AND DISCUSSION}

In order to create an out-of-plane ES structure, samples with different $\mathrm{Co} / \mathrm{Pt}$ and $\mathrm{NiFe}$ sub-units were characterized by vibrating sample magnetometry (VSM), as shown in Figure 1 . As a reference, hysteresis loops from a $[\mathrm{Co} / \mathrm{Pt}]_{10}$ sample are displayed in Figure $1 \mathrm{a}$ to confirm the perpendicular anisotropy of the film. ${ }^{44}$ Direct coupling of the $[\mathrm{Co} / \mathrm{Pt}]_{10}$ 
and $\mathrm{NiFe}(50)$ causes the sample to be magnetized almost entirely in the sample plane due to the dominant in-plane shape anisotropy of the NiFe (Figure 1c). The orientation of the magnetization of the $[\mathrm{Co} / \mathrm{Pt}] / \mathrm{NiFe}$ hybrid structure can be further modified by introducing an additional Pt spacer layer, which reduces the strength of the coupling between the sub-units (Figure 1 b). Finally, the orientation of the magnetization of the hybrid structure can be manipulated be changing the thickness $d$ of the NiFe layer. It is expected that upon decreasing the NiFe thickness, the competition between out-of-plane and in-plane anisotropy should result in gradual tilting of the magnetization, as previously observed in similar $[\mathrm{Co} / \mathrm{Pd}] / \mathrm{NiFe},{ }^{27,45}[\mathrm{Co} / \mathrm{Pd}] / \mathrm{CoFeB}^{11}$ and $\mathrm{Lr}_{1}-\mathrm{CoPt} / \mathrm{NiFe}^{29}$ structures. Indeed, shown in Figure $1 d$, a canted magnetic state is evident in the case of the $[\mathrm{Co} / \mathrm{Pt}]_{10} / \mathrm{NiFe}(10)$ sample, i.e., for the thinnest $\mathrm{NiFe}$ layer studied.
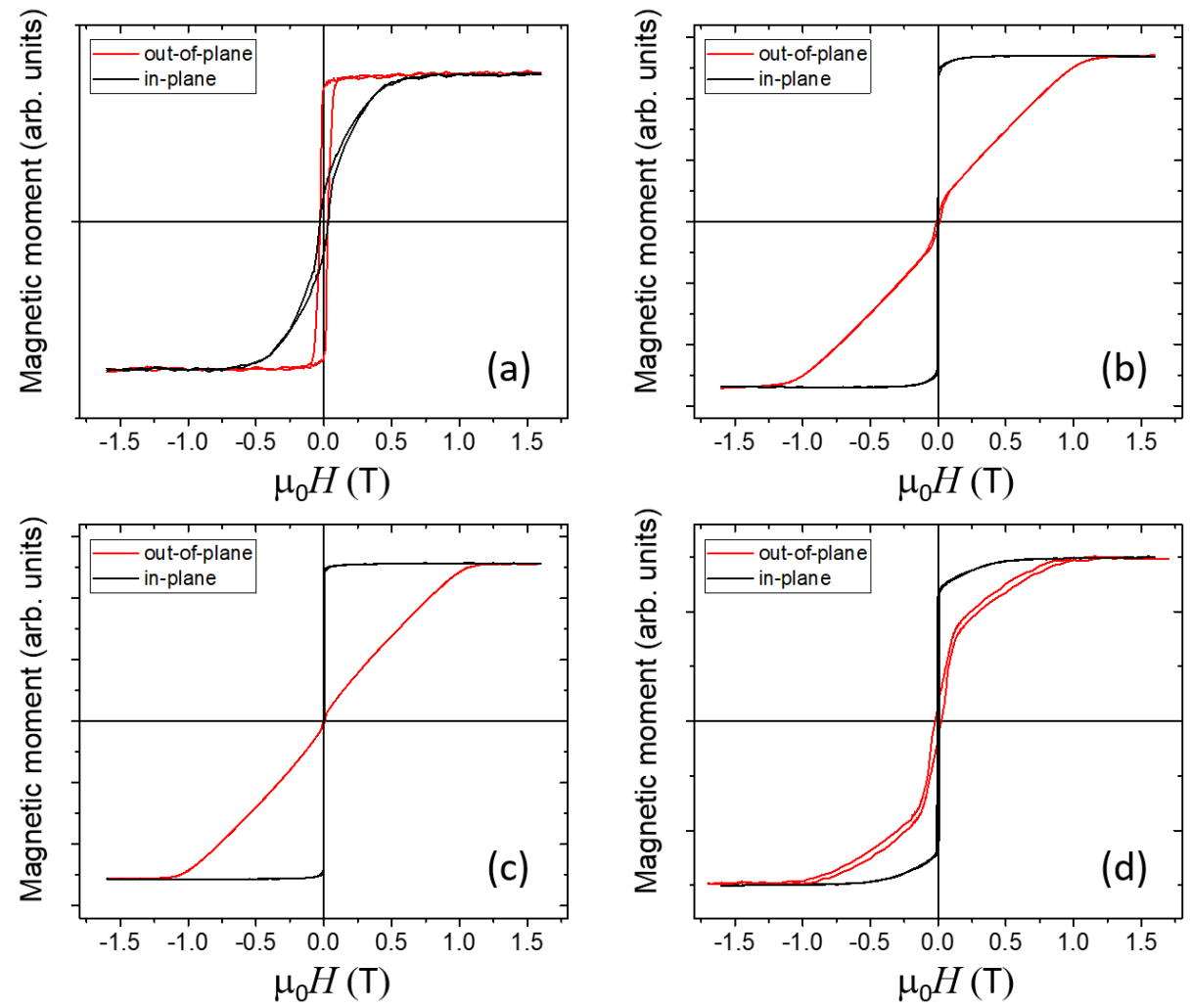

Figure 1: Vibrating-sample magnetometry (VSM) hysteresis loops acquired with the field applied in-plane (black lines) and out-of-plane (red lines) for: (a) $[\mathrm{Co} / \mathrm{Pt}]_{10}$ reference sample; (b) $[\mathrm{Co} / \mathrm{Pt}]_{10} / \mathrm{Pt}(1.5) / \mathrm{NiFe}(50) ;(\mathrm{c})[\mathrm{Co} / \mathrm{Pt}]_{10} / \mathrm{NiFe}(50)$; and (d) $[\mathrm{Co} / \mathrm{Pt}]_{10} /$ $\mathrm{NiFe}(10)$. 
Vector network analyzer ferromagnetic resonance (VNA-FMR) measurements were next carried out to investigate the effect of different coupling between the $[\mathrm{Co} / \mathrm{Pt}]$ and NiFe sub-units on their FMR properties. Maps of the scattering matrix parameter $\left|S_{12}\right|$ for the bias field applied in the sample plane and perpendicular to the sample plane are shown in Figure 2a-e and Figure 2f, respectively, for different values of $d$. For comparison, data for a sample with an additional Pt spacer layer $[\mathrm{Co} / \mathrm{Pt}]_{10} / \mathrm{Pt}(3) / \mathrm{NiFe}(50)$ is shown in Figure 2a. The $3 \mathrm{~nm}$ thick spacer layer almost completely decouples the $[\mathrm{Co} / \mathrm{Pt}]$ and $\mathrm{NiFe}$ and the FMR closely resembles that of a single NiFe layer ${ }^{46-49}$ with resonance frequency $f$ that follows a typical Kittel-like curve $f=\gamma \mu_{0} \sqrt{\left(H+H_{u}\right)\left(H+H_{u}+M_{s}\right)}, 47,50$ plotted in Figure 2a as a dashed red line (where $\gamma=29.4 \mathrm{GHz} / \mathrm{T}$ is the gyromagnetic ratio, and $\mu_{0} \mathrm{H}, \mu_{0} M_{s}=1 \mathrm{~T}$, and $\mu_{0} H_{u}=0.002 \mathrm{~T}$ are the applied, demagnetizing, and in-plane anisotropy induction fields respectively). The FMR of decoupled $[\mathrm{Co} / \mathrm{Pt}]$ is out of the range of the available frequency/field ${ }^{51}$ and so could not be captured in Figure $2 \mathrm{a}$. In the $[\mathrm{Co} / \mathrm{Pt}]_{10} / \mathrm{NiFe}(50)$ sample shown in Figure $2 \mathrm{~b},[\mathrm{Co} / \mathrm{Pt}]$ and $\mathrm{NiFe}$ are directly coupled, leading to an increase of the FMR linewidth. For the case of direct exchange coupling, the magnetization of the $[\mathrm{Co} / \mathrm{Pt}]$ lies close to the film plane, reducing its precession frequency. One can observe a characteristic splitting of the resonance at around $7 \mathrm{GHz}$, which deviates from the Kittel-like curve above ${ }_{7} \mathrm{GHz}$, with an apparent upward shift in frequency. Such behavior has been previously observed for $\mathrm{Co} / \mathrm{NiFe}$ structures and attributed to hybridization of 'optic' and 'acoustic' coupled modes, ${ }^{52,53}$ where the moments of the two layers precess in anti-phase and in-phase, respectively.

The in-plane VNA-FMR frequency vs field maps for directly coupled layers with NiFe thicknesses of 30, 20 and $10 \mathrm{~nm}$ are shown in Figure $2 \mathrm{c}, \mathrm{d}$ and e, respectively. The FMR linewidth increases substantially with decreasing NiFe thickness, with the FMR line becoming increasingly blurred above ${ }_{4} \mathrm{GHz}$ for the $\mathrm{NiFe}(30)$ and $\mathrm{NiFe}(20)$ samples. For the $[\mathrm{Co} / \mathrm{Pt}]_{10} / \mathrm{NiFe}(10)$ sample, the FMR signal almost completely disappears and 
(a)

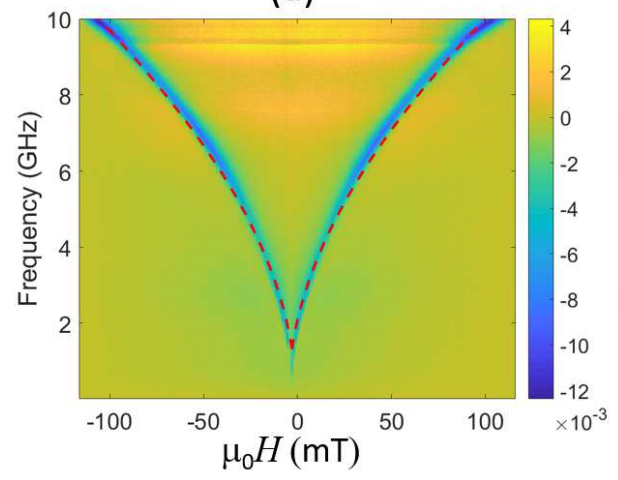

(c)

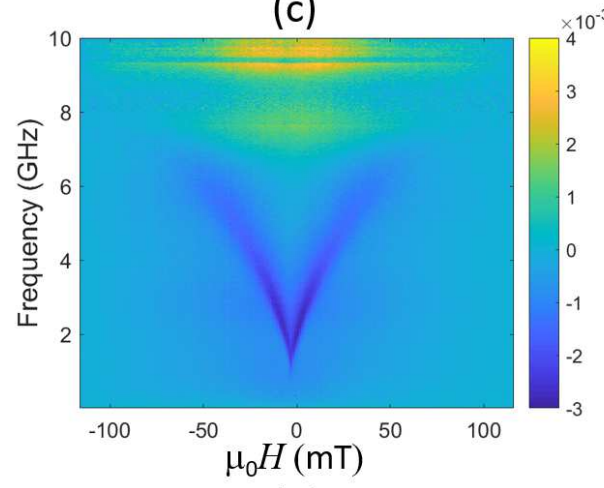

(e)

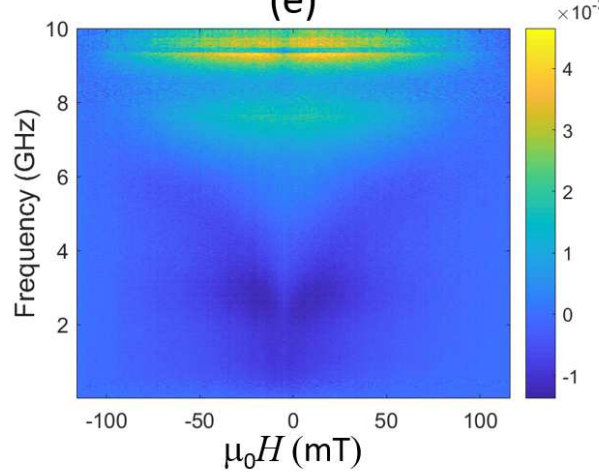

(b)

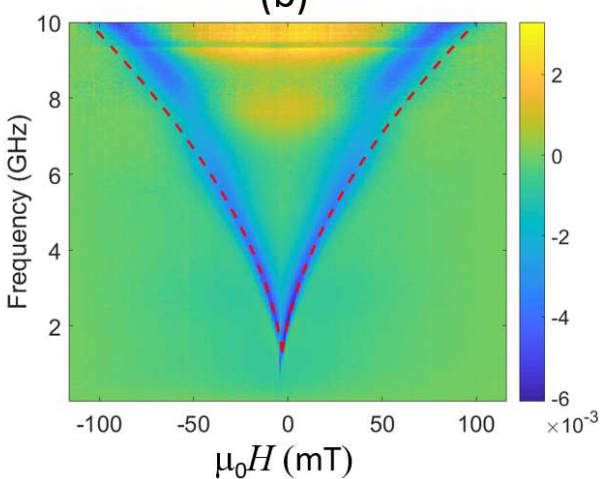

(d)

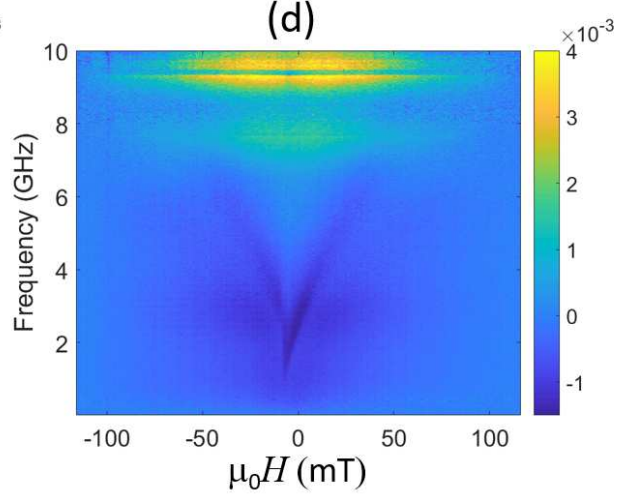

(f)

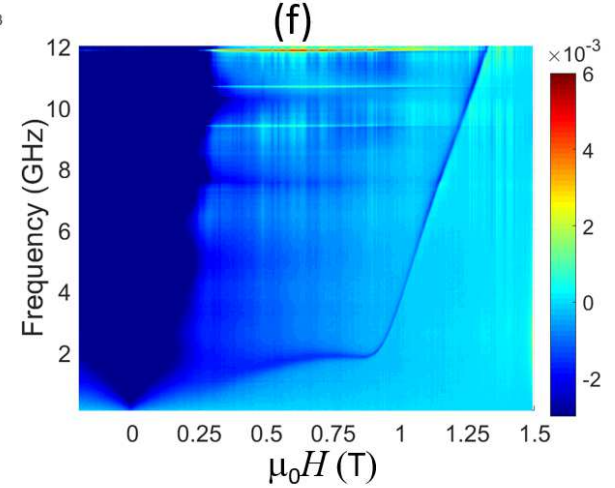

Figure 2: VNA-FMR frequency vs field maps for (a) $[\mathrm{Co} / \mathrm{Pt}]_{10} / \mathrm{Pt}(3) / \mathrm{NiFe}(50)$, (b) $[\mathrm{Co} / \mathrm{Pt}]_{10} / \mathrm{NiFe}(50),(\mathrm{c})[\mathrm{Co} / \mathrm{Pt}]_{10} / \mathrm{NiFe}(30),(\mathrm{d})[\mathrm{Co} / \mathrm{Pt}]_{10} / \mathrm{NiFe}(20)$ and $(\mathrm{e}, \mathrm{f})[\mathrm{Co} / \mathrm{Pt}]_{10} /$ $\mathrm{NiFe}(10)$ structures. The magnetic field was applied in the sample plane (a-e) and out-ofplane (f), respectively. The color scale represent the magnitude of the complex scattering matrix parameter $\left|S_{12}\right|$. The magnetic field was swept from positive towards negative values. 
only a weak, broad resonance in the $1-4 \mathrm{GHz}$ frequency range can be distinguished (Figure 2e). This indicates a very large damping, which is difficult to quantify due to the lack of a well defined FMR lineshape. The observed VNA-FMR cannot be explained solely by effects associated with reduced NiFe thickness, e.g., the two-magnon scattering mechanism due to interface roughness. ${ }^{54,55}$ Even for NiFe thicknesses of $5 \mathrm{~nm}$, a clear FMR signal should be observable for the full range of frequencies studied.47,54 It is most likely that increased damping is associated with the non-uniform magnetic configuration resulting from the coupling between the $[\mathrm{Co} / \mathrm{Pt}]$ and NiFe. Upon decreasing the NiFe thickness, the competition between out-of-plane and in-plane anisotropy should result in gradual tilting of the interfacial spins, as previously observed in similar $[\mathrm{Co} / \mathrm{Pd}] / \mathrm{NiFe}^{27,45}[\mathrm{Co} / \mathrm{Pd}] / \mathrm{CoFeB}^{11}$ and $\mathrm{L}_{1}-\mathrm{CoPt} / \mathrm{NiFe}^{29}$ structures. Figure $2 \mathrm{f}$ shows the VNA-FMR for a NiFe thickness of 1o nm (the same as in Figure 1e) but for the field applied perpendicular to the sample plane. Above the saturation induction of $0.9 \mathrm{~T}$, the expected linear dependence of FMR frequency upon field is observed. Below $0.9 \mathrm{~T}$, a broadened FMR can be distinguished down to around $0.5 \mathrm{~T}$. As for the case of in-plane VNA-FMR, an increased FMR linewidth is associated with a non-uniform magnetization. The strong absorption below $0.25 \mathrm{~T}$ is a background signal arising from the coplanar waveguide (CPW). The existence and shape of the FMR line below 0.9 T is particularly sensitive to the exact field alignment and will be the focus of a future publication.

As shown in Figure 1 and Figure 2, both VSM hysteresis loops and VNA-FMR indicate a canted magnetic state in the case of the $[\mathrm{Co} / \mathrm{Pt}]_{10} / \mathrm{NiFe}(10)$ sample. To gain more insight into the orientation of the magnetization within the hybrid structure, elementspecific $x$-ray magnetic circular dichroism $(X M C D)$ hysteresis loops were measured with the magnetic field applied along the sample normal and at a grazing angle of $30^{\circ}$, as shown in Figure $3 a$ and $3 b$. The XMCD signal was detected in transmission via $x$-ray excited optical luminescence in the $\mathrm{Al}_{2} \mathrm{O}_{3}$ substrate. The comparison of $\mathrm{Co}$ and $\mathrm{Fe}$ mag- 

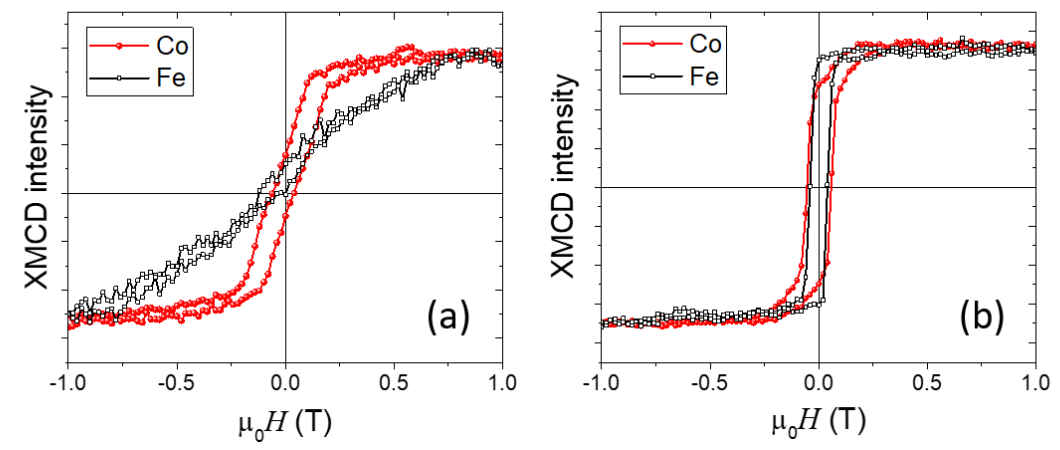

Figure 3: Normalized XMCD hysteresis loops for the $[\mathrm{Co} / \mathrm{Pt}]_{10} / \mathrm{NiFe}(10)$ sample, acquired at the $\mathrm{Co}$ (red) and Fe (black) $L_{3}$ absorption edges, with the magnetic field applied (a) along the sample normal, and (b) at a grazing angle of $30^{\circ}$ with respect to the the sample plane.

netization reversal in both geometries suggests that the Fe moments have a smaller outof-plane component and are mainly oriented in the sample plane, while the Co moments have a larger out-of-plane component and reduced (with respect to Fe) in-plane component. Hence, it is reasonable to assume that the magnetization is canted mostly within the $[\mathrm{Co} / \mathrm{Pt}]$ and $[\mathrm{Co} / \mathrm{Pt}] / \mathrm{NiFe}$ interfacial region. The orientation of $\mathrm{Co}$ and Fe moments is different due to the competition between the in-plane anisotropy of NiFe and perpendicular anisotropy of $[\mathrm{Co} / \mathrm{Pt}]$ and the exchange coupling across the interface between the two sub-units.

Since XMCD probes the weighted signal from the $[\mathrm{Co} / \mathrm{Pt}]$, we cannot distinguish between a uniformly canted magnetization and a depth/thickness dependent non-collinear magnetization distribution with varying tilting angle through the $[\mathrm{Co} / \mathrm{Pt}]$ multilayer. Determination of the depth profile of the magnetization requires more sophisticated techniques such as polarized neutron reflectometry ${ }^{56}$ or x-ray resonant magnetic reflectivity. ${ }^{57}$ Although simulations of the hysteresis loops can provide some insight, crude assumptions are often needed to estimate the tilting angle of the magnetization, ${ }^{31}$ so that it is difficult to account for effects associated with the formation of a domain structure. As shown previously for $[\mathrm{Co} / \mathrm{Pd}] / \mathrm{NiFe}^{41}$ directly coupled hard and soft sub-units result in magnetic hybrid structures with unique properties, such as imprinted magnetiza- 
tion textures and three dimensional canting of the magnetization through the thickness of the structure, which would not occur in single layer films. Imprinted magnetization structures lead to complex magnetic domains mediated through the interfacial exchange interaction that can be responsible for increased damping, as observed by VNA-FMR in Figure $2 \mathrm{~d}$,e. The remainder of this article focuses on $[\mathrm{Co} / \mathrm{Pt}]_{10} / \mathrm{NiFe}(10)$, where a canted state of magnetization within the $[\mathrm{Co} / \mathrm{Pt}]$ has been established and for which the exchange coupling between the layers appears to have the most pronounced effect on their magnetization dynamics (as shown in Figure 2).

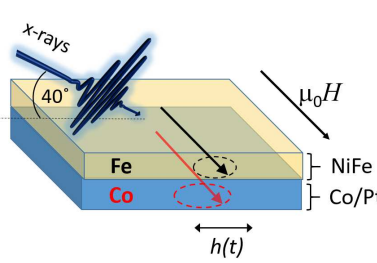

(a)

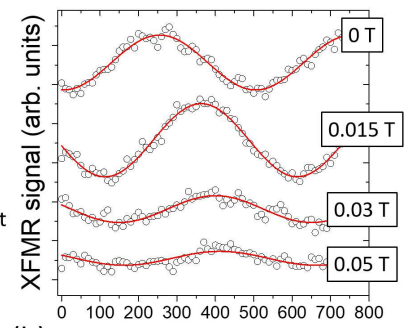

(b)
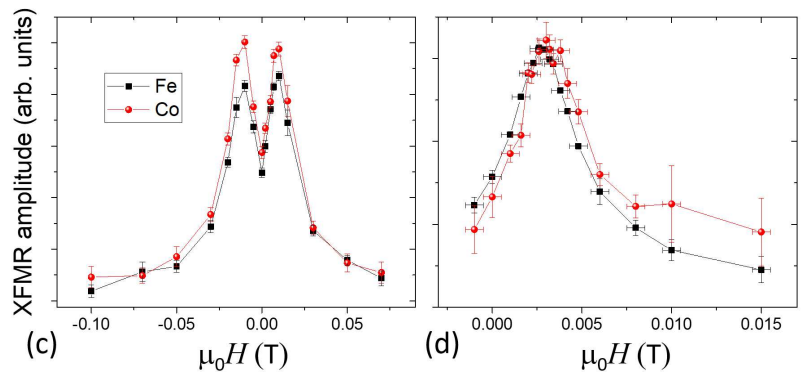

Figure 4: (a) Schematic of the experimental geometry for XFMR measurements. Precession of the magnetization about the bias induction field $\mu_{0} \mathrm{H}$ is induced by an in-plane RF magnetic field $h(t)$ oscillating at a frequency of $2 \mathrm{GHz}$. The x-ray beam is incident at a grazing angle of $40^{\circ}$ with respect to the sample plane. By tuning the x-ray energy, the magnetization precession of $\mathrm{Co}$ and Fe can be probed selectively. (b) XFMR delay scans for $\mathrm{Co}$ at different bias fields for $[\mathrm{Co} / \mathrm{Pt}]_{10} / \mathrm{NiFe}(10)$. Normalized amplitude of Fe and Co moment precession for (c) $[\mathrm{Co} / \mathrm{Pt}]_{10} / \mathrm{NiFe}(10)$, and (d) $[\mathrm{Co} / \mathrm{Pt}]_{10} / \mathrm{NiFe}(50)$.

By tuning the x-ray energy to the absorption edge of the element of interest, the XFMR signals from the $\mathrm{NiFe}\left(\mathrm{Fe} L_{3}\right.$ edge) and $\left[\mathrm{Co} / \mathrm{Pt}\right.$ ( $\mathrm{Co} L_{3}$ edge) were detected separately, allowing direct measurement of the dynamics within each layer, as shown schematically in Figure 4a. Both the amplitude and phase of precession were extracted by fitting a sine wave to the XFMR delay scans. In Figure $4 b$, representative delay scans (black circles) and their sine wave fits (red lines) are shown for different bias fields. The amplitudes of the Fe and Co moments, normalized to their static XMCD signals, are shown in Figure $4 \mathrm{C}$ and are generally similar. Interestingly, at the peaks that indicate the center of the resonance, the precession amplitude is somewhat larger for Co than for $\mathrm{Fe}$, 
implying that the $[\mathrm{Co} / \mathrm{Pt}]$ magnetization precesses with larger cone angle than the $\mathrm{NiFe}$ magnetization. For comparison, the amplitudes obtained from $[\mathrm{Co} / \mathrm{Pt}]_{10} / \mathrm{NiFe}(5 \mathrm{O})$ are shown in Figure $4 \mathrm{~d}$. Here, both layers precess with the same normalized amplitude, as expected for directly coupled layers. The precession amplitude is thus different for the two coupled layers only when the magnetization of both layers is canted. For both samples, the $[\mathrm{Co} / \mathrm{Pt}]$ and $\mathrm{NiFe}$ layers precess in-phase, as again expected for directly coupled ferromagnetic layers (see Figure $S_{1}$ in the Supporting Information showing the phase of the magnetization precession). Note that the XFMR linewidth for the $[\mathrm{Co} / \mathrm{Pt}]_{10} / \mathrm{NiFe}(10)$ (Figure ${ }_{4} \mathrm{C}$ ) is around five times larger in comparison to the $[\mathrm{Co} / \mathrm{Pt}]_{10} / \mathrm{NiFe}(5 \mathrm{O})$ (Figure $4 \mathrm{~d}$ ), in agreement with the VNA-FMR measurements shown in Figure 2. The XFMR signal was also detected with the field applied perpendicular to the sample plane (see Figure S2 in the Supporting Information), and the Fe and Co amplitudes were again found to be slightly different at the peak of the resonance.
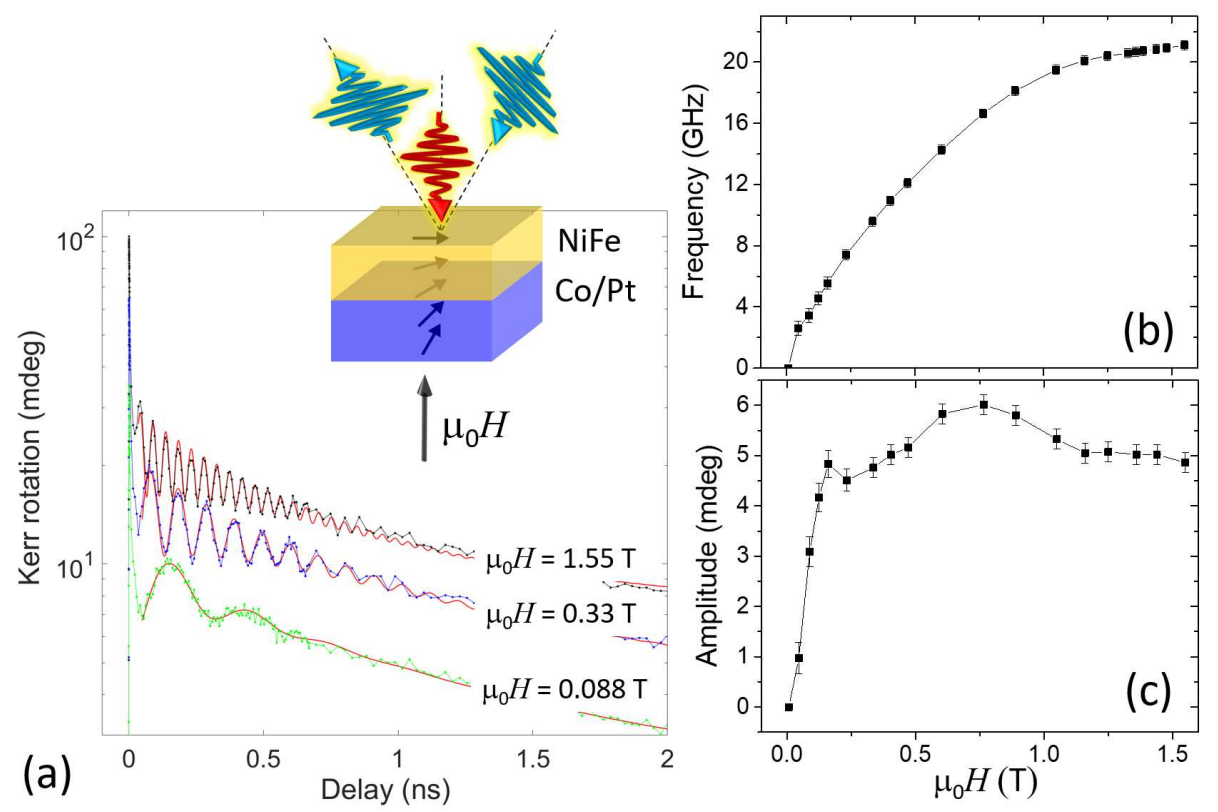

Figure 5: TRMOKE measurements made upon $[\mathrm{Co} / \mathrm{Pt}]_{10} / \mathrm{NiFe}(10)$ with a polar magnetic field and laser pump pulse energy of $0.55 \mu \mathrm{J}$ : (a) oscillatory Kerr signals for different induction field values of $\mu_{0} \mathrm{H}$; dependence of (b) oscillation frequency and (c) amplitude upon $\mu_{0} H$. The inset to (a) shows a schematic of the TRMOKE experiment and the magnetization profile for small field values. 
To further explore the effect of the canted state upon the magnetization dynamics, TRMOKE measurements were performed on the $[\mathrm{Co} / \mathrm{Pt}]_{10} / \mathrm{NiFe}(10)$ sample with a polar field. Figure 5a shows TRMOKE delay scans obtained for different values of the bias induction field $\mu_{0} H$ with the laser pump pulse energy fixed at $0.55 \mu \mathrm{J}$. The abrupt initial rise of the signal is associated with the optically induced ultrafast demagnetization, during which the saturation magnetization and magnetic anisotropy are reduced as the electron and spin temperatures rise. ${ }^{58,59}$ An oscillatory Kerr signal is then observed due to precession of the magnetization. 59 The signal was fitted to the sum of a decaying exponential and a damped sinusoid, of form $A \exp (-B t)+C \sin (2 \pi f t+\phi) \exp (-t / \tau)$, where the relevant fitting parameters are $C, f, \tau$ and $\phi$, corresponding to the initial amplitude, frequency, relaxation rate, and initial phase of the precessional signal. The fitted curves successfully reproduce the oscillatory Kerr signal (see Figure 5a) and allow the frequency and amplitude of precession to be extracted, which are plotted in Figure $5 \mathrm{~b}$ and c, respectively. The existence of the oscillatory signal at magnetic induction values above $\mathrm{IT}$ is a key finding of this article. For a single layer film with in-plane remanent magnetization, no precession occurs when $\mu_{0} H$ is larger than the polar saturation field (about $0.9 \mathrm{~T}$ in the present case) because the total effective magnetic field acting upon the magnetization lies normal to the plane, and hence parallel to the magnetization, both before and after optical pumping. The presence of magnetization precession at such high magnetic fields suggests that the sample $[\mathrm{Co} / \mathrm{Pt}]_{10} / \mathrm{NiFe}(\mathrm{IO} \mathrm{nm})$ exhibits a mutually imprinted domain structure, as previously observed in $[\mathrm{Co} / \mathrm{Pt}] / \mathrm{NiFe}^{60,61}$ and $[\mathrm{Co} / \mathrm{Pd}] / \mathrm{NiFe} .{ }^{11,42}$ Variations in structure, composition and morphology act to stabilize a domain structure that, in concert with the abrupt transition from in-plane to out-ofplane anisotropy at the interface between the $[\mathrm{Co} / \mathrm{Pt}]$ and $\mathrm{NiFe}$, can cause the canted magnetization profile to become locked. This leads to a more complex process of magnetization reversal compared to a single layer thin film, with much larger magnetic fields being required to achieve full technical saturation. Hence, even at the highest available 
field of $1.5 \mathrm{~T}$, torque on canted magnetic moments in the vicinity of the interface is responsible for driving the optically induced magnetization precession. This idea is reinforced by the observation that the amplitude of precession is fairly constant over a wide field range (Figure $5 \mathrm{c}$ ) for which the orientation of the locked interfacial moments remains largely unaltered. A steep increase of the amplitude up to $0.2 \mathrm{~T}$ can be correlated with reorientation of the $\mathrm{Co} / \mathrm{Pt}$ sub-unit magnetization towards the sample normal direction, as can be deduced from the out-of-plane hysteresis loops (Figure $1 \mathrm{~d}$ and Figure 3a). Furthermore, as can be observed in Figure $1 \mathrm{~d}$ and Figure 3a, the magnetization seems to saturate around $1 \mathrm{~T}$ and further increase of the magnetic field does not result in any noticeable change in the magnetization. This behavior is also reflected in the dynamic response in Figure $5 \mathrm{~b}$, where the frequency vs field curve appears to saturate above $1 \mathrm{~T}$, which supports the idea that the oscillatory signal originates mainly from the interfacial $[\mathrm{Co} / \mathrm{Pt}] \mathrm{NiFe}$ moments, the orientation of which remains largely unaltered by further increase of the applied field. In contrast, the signal observed in VNA-FMR experiments arises from the microwave field acting on the spatially averaged magnetization, that is dominated by NiFe moments with essentially uniform magnetic configuration. The different origin of the precession is reflected in the very different frequencies observed in the two experiments, e.g., at $1 \mathrm{~T}$ in the out-of-plane geometry, where all moments are expected to be aligned with the applied field, the resonance frequency is $4 \mathrm{GHz}$ in the VNA-FMR (Figure 2f) but ${ }_{19} \mathrm{GHz}$ frequency in the TRMOKE (Figure $5 \mathrm{~b}$ ). A comparison of effective damping parameters $\alpha_{\text {eff }}$ estimated from TRMOKE with $\alpha_{\text {eff }}=1 /(2 \pi f \tau)$, and VNA-FMR with $\alpha_{\text {eff }}=\Delta H \gamma /(4 \pi f)$ (where $\Delta H$ is the FMR linewidth and $\gamma$ is the gyromagnetic ratio), actually yields similar values above the expected saturation field, even though the resonance frequencies are quite different (see Figure $S_{3}$ in the Supporting Information).

The amplitude and relaxation time of the oscillatory Kerr signal (Figure $S_{4}$ in the Supporting Information) depends upon the pump laser fluence, suggesting that further 
control of the magnetization dynamics associated with the imprinted domain structure might be possible. The canted magnetization studied here could be exploited within optically stimulated spin valves, where an ultrafast spin current is transmitted between two ferromagnetic layers via spin polarized hot electrons. ${ }^{23,25,62,63}$ By tuning the orientation of the source and/or sink layer magnetization, greater control could be exerted over the transverse component of the spin current and hence the STT acting on the sink layer. In addition, non-collinear spin structures such as $[\mathrm{Co} / \mathrm{Pt}] / \mathrm{NiFe}$ exchange springs could allow for more versatile manipulation of the spin polarization of hot electrons. ${ }^{25}$ Finally, the ability to maintain the precession at high magnetic fields might be an asset to future magnonic devices, making them more robust against external perturbations.

\section{CONCLUSIONS}

In summary, a non-collinear magnetization structure was stabilized by exchange coupling a $[\mathrm{Co} / \mathrm{Pt}]$ multilayer with perpendicular anisotropy to a NiFe layer with in-plane anisotropy. The comparison between microwave and optically induced magnetization dynamics suggests that a mutually imprinted magnetic domain configuration is formed. The imprinted domains cause canted interfacial moments to remain locked, even in the presence of large static magnetic fields, and allow optically stimulated magnetization precession to be observed for an extended magnetic field and frequency range. The ability to tune the canting of the magnetization of $[\mathrm{Co} / \mathrm{Pt}] / \mathrm{NiFe}$ hybrid structures may allow for greater control of optically excited magnetization reversal and the transfer of angular momentum by laser-excited hot electrons. Magnetic hybrid structures of the kind studied here therefore have great potential for practical applications in both microwaveassisted magnetic recording (MAMR) and heat-assisted magnetic recording (HAMR). 


\section{EXPERIMENTAL SECTION}

Sample preparation and characterization. $[\mathrm{Co}(0.4 \mathrm{~nm}) / \mathrm{Pt}(0.9 \mathrm{~nm})]_{10} / \mathrm{Ni}_{81} \mathrm{Fe}_{19}(d)$ films with $\mathrm{Ni}_{81} \mathrm{Fe}_{19}$ layer thickness $d$ were deposited on $\mathrm{Al}_{2} \mathrm{O}_{3}$ substrates using dc magnetron sputtering. The films were grown on a $\mathrm{Pt}(30 \mathrm{~nm})$ seed layer and capped with a $\mathrm{Pt}(3 \mathrm{~nm})$ protective layer. Hysteresis loops were measured in a vibrating sample magnetometer (VSM) and layer thicknesses were verified by x-ray reflectometry (XRR). Element-specific x-ray magnetic circular dichroism (XMCD) hysteresis loops were measured via $\mathrm{x}$-ray excited optical luminescence in the $\mathrm{Al}_{2} \mathrm{O}_{3}$ substrate on beamline 6.3.1 of the Advanced Light Source (ALS) to probe the magnetization reversal of the $[\mathrm{Co} / \mathrm{Pt}]$ and NiFe independently.

Vector network analyzer ferromagnetic resonance (VNA-FMR) measurements. The VNA-FMR measurements were performed on a standard coplanar waveguide (CPW) with $50 \Omega$ impedance and with a signal line of $500 \mu \mathrm{m}$ width. An electromagnet mounted on a rotating stage supplied a bias magnetic field either in the sample plane or normal to the sample plane. The measurements were performed by stepping magnetic field values and sweeping the microwave field within the frequency range o-20 GHz. The sample was placed face down, with the magnetic layers in close proximity to the waveguide. For each bias field value a reference measurement was made at high field and subtracted in order to remove the background signal originating from the CPW and coaxial cables.

X-ray ferromagnetic resonance (XFMR) experiments. XFMR ${ }^{37-40}$ measurements were carried out on beamlines 4.0.2 of the Advanced Light Source (ALS) and I1o at the Diamond Light Source (DLS), by monitoring absorption/transmission as a function of time delay between a synchronized RF magnetic field (pumping the spin precession) and circularly polarized $x$-ray pulses (probing the oscillatory magnetization component along the x-ray wavevector). The $x$-rays were incident at a grazing angle of $40^{\circ}$. The sample was placed 
face down on a coplanar waveguide (CPW) with a countersunk hole of $500 \mu \mathrm{m}$ diameter allowing the incident $x$-ray beam to access the surface of the sample, while the transmitted $\mathrm{x}$-rays were converted to optical radiation through $\mathrm{x}$-ray excited optical luminescence in the $\mathrm{Al}_{2} \mathrm{O}_{3}$ substrate, with the emitted light detected by a photodiode mounted behind the sample.

Time resolved magneto-optical Kerr effect (TRMOKE) measurements. Optically induced magnetization dynamics were probed by TRMOKE measurements, using an amplified Ti:sapphire pulsed laser source with $20 \mathrm{kHz}$ repetition rate and $50 \mathrm{fs}$ pulse width. The spolarized $800 \mathrm{~nm}$ pump and p-polarized $400 \mathrm{~nm}$ probe beams were incident normal and at $10^{\circ}$ from normal to the sample plane, respectively, and focused to $90 \mu \mathrm{m}$ and $25 \mu \mathrm{m}$ full width at half maximum spot diameters. The measured Kerr rotation is predominantly due to the out-of-plane component of the magnetization via the polar MOKE. All measurements presented in this work were performed at room temperature.

\section{Supporting Information Available}

The following files are available free of charge.

- Supporting Information: Vibrating-sample magnetometry (VSM) hysteresis loops, additional $\mathrm{x}$-ray ferromagnetic resonance $(\mathrm{XFMR})$ and time-resolved magnetooptical Kerr effect (TRMOKE) results.

\section{Acknowledgement}

The authors acknowledge the Engineering and Physical Sciences Research Council (EPSRC) under Grant Numbers EP/Po21190/1, EP/Po20151/1, and EP/Po2047X/1. Beamtime awarded on I1o at the Diamond Light Source (SI17745-1, SI19116-1 and SI20760-1) 
is acknowledged. This research used resources of the Advanced Light Source, which is a DOE Office of Science User Facility under contract no. DE-AC02-05CH11231. D.G.N. acknowledges support via the EPSRC Centre for Doctoral Training in Metamaterials (Grant No. EP/Lo15331/1).

\section{Author Contributions}

M.D., T.H., G.v.d.L. and R.J.H. conceived the study. M.D. fabricated the samples. M.D., A.F., D.M.B., D.G.N., C.K., A.T.N., E.A. and P.S. performed measurements and contributed to the data analysis. M.D. and R.J.H. prepared the manuscript with the help of G.J.B., T.H., G.v.d.L. and G.H. All authors discussed the results and commented on the manuscript.

\section{Notes}

The authors declare no competing financial interest.

\section{References}

(1) Gutfleisch, O.; Willard, M. A.; Brück, E.; Chen, C. H.; Sankar, S. G.; Liu, J. P. Magnetic Materials and Devices for the 21st Century: Stronger, Lighter, and More Energy Efficient. Adv. Mater. 2011, 23, 821-842.

(2) Fullerton, E. E.; Jiang, J. S.; Bader, S. D. Hard/soft magnetic heterostructures: model exchange-spring magnets. J. Magn. Magn. Mater. 1999, 200, 392-404.

(3) Seki, T.; Utsumiya, K.; Nozaki, Y.; Imamura, H.; Takanashi, K. Spin wave-assisted reduction in switching field of highly coercive iron-platinum magnets. Nat. Commun. 2013, 4, 1726. 
(4) Shelford, L. R.; Liu, Y.; Al-Jarah, U.; de Groot, P. A. J.; Bowden, G. J.; Ward, R. C. C.; Hicken, R. J. Ultrafast Optical Parametric Pumping of Magnetization Reorientation and Precessional Dynamics in $\mathrm{DyFe}_{2} / \mathrm{YFe}_{2}$ Exchange Springs. Phys. Rev. Lett. 2014, 113, 067601.

(5) Zhu, J.; Zhu, X.; Tang, Y. Microwave Assisted Magnetic Recording. IEEE Trans. Magn. 2008, 44, 125-131.

(6) Challener, W. A.; Peng, C.; Itagi, A. V.; Karns, D.; Peng, W.; Peng, Y.; Yang, X.; Zhu, X.; Gokemeijer, N. J.; Hsia, Y.-T.; Ju, G.; Rottmayer, R. E.; Seigler, M. A.; Gage, E. C. Heat-assisted magnetic recording by a near-field transducer with efficient optical energy transfer. Nat. Photonics 2009, 3, 220-224.

(7) Kneller, E. F.; Hawig, R. The exchange-spring magnet: a new material principle for permanent magnets. IEEE Trans. Magn. 1991, 27, 3588-3560.

(8) Dürr, H. A.; Guo, G. Y.; van der Laan, G.; Lee, J.; Lauhoff, G.; Bland, J. A. C. ElementSpecific Magnetic Anisotropy Determined by Transverse Magnetic Circular X-ray Dichroism. Science 1997, 277, 213.

(9) Stenning, G. B. G.; Bowden, G. J.; Gregory, S. A.; de Groot, P. A. J.; van der Laan, G.; Shelford, L. R.; Bencok, P.; Steadman, P.; Dobrynin, A. N.; Hesjedal, T. Transverse magnetic exchange springs in a $\mathrm{DyFe}_{2} / \mathrm{YFe}_{2}$ superlattice. Phys. Rev. B 2012, 86, 174420.

(10) Tacchi, S.; Nguyen, T. N. A.; Carlotti, G.; Gubbiotti, G.; Madami, M.; Dumas, R. K.; Lau, J. W.; Åkerman, J.; Rettori, A.; Pini, M. G. Spin wave excitations in exchangecoupled $[\mathrm{Co} / \mathrm{Pd}]-\mathrm{NiFe}$ films with tunable tilting of the magnetization. Phys. Rev. B $2013,87,144426$.

(11) Nguyen, T. N. A.; Knut, R.; Fallahi, V.; Chung, S.; Le, Q. T.; Mohseni, S. M.; Karis, O.; 
Peredkov, S.; Dumas, R. K.; Miller, C. W.; Åkerman, J. Depth-Dependent Magnetization Profiles of Hybrid Exchange Springs. Phys. Rev. Appl. 2014, 2, 044014.

(12) Zhou, Y.; Zha, C. L.; Bonetti, S.; Persson, J.; Åkerman, J. Spin-torque oscillator with tilted fixed layer magnetization. Appl. Phys. Lett. 2008, 92, 262508.

(13) Zhang, H.; Lin, W.; Mangin, S.; Zhang, Z.; Liu, Y. Signature of magnetization dynamics in spin-transfer-driven nanopillars with tilted easy axis. Appl. Phys. Lett. 2013, 102, 012411.

(14) Fallarino, L.; Sluka, V.; Kardasz, B.; Pinarbasi, M.; Berger, A.; Kent, A. D. Interlayer exchange coupling between layers with perpendicular and easy-plane magnetic anisotropies. Appl. Phys. Lett. 2016, 109, 082401.

(15) You, L.; Lee, O.; Bhowmik, D.; Labanowski, D.; Hong, J.; Bokor, J.; Salahuddin, S. Switching of perpendicularly polarized nanomagnets with spin orbit torque without an external magnetic field by engineering a tilted anisotropy. Proc. Natl. Acad. Sci. U. S. A. 2015, 112, 10310-10315.

(16) Tserkovnyak, Y.; Brataas, A.; Bauer, G. E. W. Spin pumping and magnetization dynamics in metallic multilayers. Phys. Rev. B 2002, 66, 224403.

(17) Woltersdorf, G.; Mosendz, O.; Heinrich, B.; Back, C. H. Magnetization Dynamics due to Pure Spin Currents in Magnetic Double Layers. Phys. Rev. Lett. 2007, 99, 246603 .

(18) Sankey, J. C.; Cui, Y.-T.; Sun, J. Z.; Slonczewski, J. C.; Buhrman, R. A.; Ralph, D. C. Measurement of the spin-transfer-torque vector in magnetic tunnel junctions. Nat. Phys. 2008, 4, 67-71.

(19) Kajiwara, Y.; Harii, K.; Takahashi, S.; Ohe, J.; Uchida, K.; Mizuguchi, M.; Umezawa, H.; Kawai, H.; Ando, K.; Takanashi, K.; Maekawa, S.; Saitoh, E. Trans- 
mission of electrical signals by spin-wave interconversion in a magnetic insulator. Nature 2010, 464, 262.

(20) Wei, D.; Obstbaum, M.; Ribow, M.; Back, C. H.; Woltersdorf, G. Spin Hall voltages from a.c. and d.c. spin currents. Nat. Commun. 2014, 5, 3768.

(21) Ando, K. Dynamical generation of spin currents. Semicond. Sci. Technol. 2014, 29, 043002.

(22) Baker, A. A.; Figueroa, A. I.; Love, C. J.; Cavill, S. A.; Hesjedal, T.; van der Laan, G. Anisotropic Absorption of Pure Spin Currents. Phys. Rev. Lett. 2016, 116, 047201.

(23) Schellekens, A. J.; Kuiper, K. C.; de Wit, R. R. J. C.; Koopmans, B. Ultrafast spintransfer torque driven by femtosecond pulsed-laser excitation. Nat. Commun. 2014, 5,4333 .

(24) Bergeard, N.; Hehn, M.; Mangin, S.; Lengaigne, G.; Montaigne, F.; Lalieu, M. L. M.; Koopmans, B.; Malinowski, G. Hot-Electron-Induced Ultrafast Demagnetization in Co/Pt Multilayers. Phys. Rev. Lett. 2016, 117, 147203.

(25) Alekhin, A.; Razdolski, I.; Ilin, N.; Meyburg, J. P.; Diesing, D.; Roddatis, V.; Rungger, I.; Stamenova, M.; Sanvito, S.; Bovensiepen, U.; Melnikov, A. Femtosecond Spin Current Pulses Generated by the Nonthermal Spin-Dependent Seebeck Effect and Interacting with Ferromagnets in Spin Valves. Phys. Rev. Lett. 2017, 119, 017202.

(26) Lalieu, M. L. M.; Lavrijsen, R.; Duine, R. A.; Koopmans, B. Investigating optically excited terahertz standing spin waves using noncollinear magnetic bilayers. Phys. Rev. B 2019, 99, 184439 .

(27) Nguyen, T. N. A.; Fang, Y.; Fallahi, V.; Benatmane, N.; Mohseni, S. M.; Dumas, R. K.; Åkerman, J. [Co/Pd]-NiFe exchange springs with tunable magnetization tilt angle. Appl. Phys. Lett. 2011, 98, 172502. 
(28) Banerjee, C.; Pal, S.; Ahlberg, M.; Nguyen, T. N. A.; Åkerman, J.; Barman, A. Alloptical study of tunable ultrafast spin dynamics in $[\mathrm{Co} / \mathrm{Pd}] / \mathrm{NiFe}$ systems: the role of spin-twist structure on Gilbert damping. RSC Adv. 2016, 6, 80168-80173.

(29) Saravanan, P.; Hsu, J.-H.; Tsai, C. L.; Tsai, C. Y.; Lin, Y. H.; Kuo, C. Y.; Wu, J.-C.; Lee, C.-M. Interplay between out-of-plane anisotropic L11-type CoPt and in-plane anisotropic NiFe layers in $\mathrm{CoPt} / \mathrm{NiFe}$ exchange springs. J. Appl. Phys. 2014, 115, 243905 .

(30) Saravanan, P.; Hsu, J.-H.; Chérif, S. M.; Roussigné, Y.; Belmeguenai, M.; Stashkevich, A.; Vernier, N.; Singh, A. K.; Chang, C.-R. Correlation between static and dynamic magnetic properties of highly perpendicular magnetized $\mathrm{Co}_{49} \mathrm{Pt}_{51}$ thin films. Phys. Rev. B 2015, 92, 144431.

(31) Saravanan, P.; Talapatra, A.; Mohanty, J.; Boominathasellarajan, S.; Hsu, J.-H. Study on the domain structure and tunable spin orientation in L11-CoPt/NiFe exchange springs with Ta-spacer. J. Magn. Magn. Mater. 2018, 448, 316-321.

(32) Bouloussa, H.; Roussigné, Y.; Belmeguenai, M.; Stashkevitch, A.; Hsu, J. H.; Devolder, T.; Chérif, S. M. Static and dynamic magnetic properties of $\mathrm{CoPt} / \mathrm{NiFe}$ bilayers: experiment and modelling. J. Phys. D: Appl. Phys. 2019, 53, 075001.

(33) Lambert, C.-H.; Mangin, S.; Varaprasad, B. S. D. C. S.; Takahashi, Y. K.; Hehn, M.; Cinchetti, M.; Malinowski, G.; Hono, K.; Fainman, Y.; Aeschlimann, M.; Fullerton, E. E. All-optical control of ferromagnetic thin films and nanostructures. Science 2014, 345, 1337-1340.

(34) Vomir, M.; Albrecht, M.; Bigot, J.-Y. Single shot all optical switching of intrinsic micron size magnetic domains of a Pt/Co/Pt ferromagnetic stack. Appl. Phys. Lett. 2017, 111, 242404 . 
(35) Kichin, G.; Hehn, M.; Gorchon, J.; Malinowski, G.; Hohlfeld, J.; Mangin, S. From Multiple- to Single-Pulse All-Optical Helicity-Dependent Switching in Ferromagnetic Co/Pt Multilayers. Phys. Rev. Appl. 2019, 12, 024019.

(36) Gorchon, J.; Lambert, C.-H.; Yang, Y.; Pattabi, A.; Wilson, R. B.; Salahuddin, S.; Bokor, J. Single shot ultrafast all optical magnetization switching of ferromagnetic Co/Pt multilayers. Appl. Phys. Lett. 2019, 111, 042401.

(37) Marcham, M. K.; Shelford, L. R.; Cavill, S. A.; Keatley, P. S.; Yu, W.; Shafer, P.; Neudert, A.; Childress, J. R.; Katine, J. A.; Arenholz, E.; Telling, N. D.; van der Laan, G.; Hicken, R. J. Phase-resolved x-ray ferromagnetic resonance measurements of spin pumping in spin valve structures. Phys. Rev. B 2013, 87, 180403.

(38) Li, J.; Shelford, L. R.; Shafer, P.; Tan, A.; Deng, J. X.; Keatley, P. S.; Hwang, C.; Arenholz, E.; van der Laan, G.; Hicken, R. J.; Qiu, Z. Q. Direct Detection of Pure ac Spin Current by X-Ray Pump-Probe Measurements. Phys. Rev. Lett. 2016, 117, 076602.

(39) van der Laan, G. Time-resolved X-ray detected ferromagnetic resonance of spin currents. J. Electron Spectrosc. Relat. Phenom. 2017, 220, 137-146.

(40) Klewe, C.; Li, Q.; Yang, M.; N’Diaye, A. T.; Burn, D. M.; Hesjedal, T.; Figueroa, A. I.; Hwang, C.; Li, J.; Hicken, R. J.; Shafer, P.; Arenholz, E.; van der Laan, G.; Qiu, Z. Element- and Time-Resolved Measurements of Spin Dynamics Using X-ray Detected Ferromagnetic Resonance. Synchrotron Radiat. News 2020, 33, 12-19.

(41) Bryan, M. T.; Heldt, G.; Thomson, T.; Heyderman, L. J.; Hrkac, G. Complex spin configurations in hybrid magnetic multilayer structures due to mutual spin imprinting. Phys. Rev. B 2016, 94, 104415.

(42) Heldt, G.; Bryan, M. T.; Hrkac, G.; Stevenson, S. E.; Chopdekar, R. V.; Raabe, J.; 
Thomson, T.; Heyderman, L. J. Topologically confined vortex oscillations in hybrid $[\mathrm{Co} / \mathrm{Pd}]_{8}-$ Permalloy structures. Appl. Phys. Lett. 2014, 104, 182401.

(43) Wohlhüter, P.; Bryan, M. T.; Warnicke, P.; Gliga, S.; Stevenson, S. E.; Heldt, G.; Saharan, L.; Suszka, A. K.; Moutafis, C.; Chopdekar, R. V.; Raabe, J.; Thomson, T.; Hrkac, G.; Heyderman, L. J. Nanoscale switch for vortex polarization mediated by Bloch core formation in magnetic hybrid systems. Nat. Commun. 2015, 6, 7836.

(44) Hellwig, O.; Berger, A.; Kortright, J. B.; Fullerton, E. E. Domain structure and magnetization reversal of antiferromagnetically coupled perpendicular anisotropy films. J. Magn. Magn. Mater. 2007, 319, 13-55.

(45) Tryputen, L.; Guo, F.; Liu, F.; Nguyen, T. N. A.; Mohseni, M. S.; Chung, S.; Fang, Y.; Åkerman, J.; McMichael, R. D.; Ross, C. A. Magnetic structure and anisotropy of $[\mathrm{Co} / \mathrm{Pd}]_{5} / \mathrm{NiFe}$ multilayers. Phys. Rev. B 2015, 91, 014407.

(46) Tannenwald, P. E.; Seavey, M. H. Ferromagnetic Resonance in Thin Films of Permalloy. Phys. Rev. 1957, 105, 377-378.

(47) Shaw, J. M.; Nembach, H. T.; Silva, T. J.; Boone, C. T. Precise determination of the spectroscopic g-factor by use of broadband ferromagnetic resonance spectroscopy. $J$. Appl. Phys. 2013, 114, 243906.

(48) Silva, E. F.; Corrêa, M. A.; Della Pace, R. D.; Plá Cid, C. C.; Kern, P. R.; Carara, M.; Chesman, C.; Alves Santos, O.; Rodríguez-Suárez, R. L.; Azevedo, A.; Rezende, S. M.; Bohn, F. Thickness dependence of the magnetic anisotropy and dynamic magnetic response of ferromagnetic NiFe films. J. Phys. D: Appl. Phys 2017, 50,185001 .

(49) Salikhov, R.; Alekhin, A.; Parpiiev, T.; Pezeril, T.; Makarov, D.; Abrudan, R.; Meckenstock, R.; Radu, F.; Farle, M.; Zabel, H.; Temnov, V. V. Gilbert damping in NiFeGd 
compounds: Ferromagnetic resonance versus time-resolved spectroscopy. Phys. Rev. B 2019, 99, 104412.

(50) Kittel, C. On the Theory of Ferromagnetic Resonance Absorption. Phys. Rev. 1948, 73, 155-161.

(51) Devolder, T.; Couet, S.; Swerts, J.; Kar, G. S. Gilbert damping of high anisotropy Co/Pt multilayers. J. Phys. D: Appl. Phys. 2018, 51, 135002.

(52) Crew, D. C.; Kennewell, K. J.; Lwin, M. J.; Woodward, R. C.; Prasad, S.; Stamps, R. L. Optic and acoustic modes measured in a cobalt/Permalloy exchange spring bilayer using inductive magnetometry. J. Appl. Phys. 2020, 97, 10A707.

(53) Kennewell, K. Surface and interface anisotropies measured using inductive magnetometry. PhD Thesis 2008.

(54) Rezende, S. M.; Azevedo, A.; Lucena, M. A.; de Aguiar, F. M. Anomalous spin-wave damping in exchange-biased films. Phys. Rev. B 2001, 63, 214418.

(55) Haldar, A.; Banerjee, C.; Laha, P.; Barman, A. Brillouin light scattering study of spin waves in NiFe/Co exchange spring bilayer films. J. Appl. Phys. 2014, 115, 133901.

(56) Majkrzak, C. F.; O’Donovan, K. V.; Berk, N. F.; Chatterji, T. Neutron Scattering from Magnetic Materials; Elsevier Science: Amsterdam, 2006; pp 397-471.

(57) Jal, E.; Dąbrowski, M.; Tonnerre, J. M.; Przybylski, M.; Grenier, S.; Jaouen, N.; Kirschner, J. Noncollinearity of the canted spins across ultrathin Fe films on vicinal Ag surfaces. Phys. Rev. B 2015, 91, 214418.

(58) Beaurepaire, E.; Merle, J.-C.; Daunois, A.; Bigot, J.-Y. Ultrafast Spin Dynamics in Ferromagnetic Nickel. Phys. Rev. Lett. 1996, 76, 4250-4253.

(59) Kirilyuk, A.; Kimel, A. V.; Rasing, T. Ultrafast optical manipulation of magnetic order. Rev. Mod. Phys. 2010, 82, 2731-2784. 
(6o) Kinane, C. J.; Suszka, A. K.; Marrows, C. H.; Hickey, B. J.; Arena, D. A.; Dvorak, J.; Charlton, T. R.; Langridge, S. Soft x-ray resonant magnetic scattering from an imprinted magnetic domain pattern. Appl. Phys. Lett. 2006, 89, 092507.

(61) Duckworth, T. A.; Ogrin, F. Y.; Beutier, G.; Dhesi, S. S.; Cavill, S. A.; Langridge, S.; Whiteside, A.; Moore, T.; Dupraz, M.; Yakhou, F.; van der Laan, G. Holographic imaging of interlayer coupling in Co/Pt/NiFe. New J. Phys. 2013, 15, 023045.

(62) Ritzmann, U.; Baláž, P.; Maldonado, P.; Carva, K.; Oppeneer, P. M. High-frequency magnon excitation due to femtosecond spin-transfer torques. Phys. Rev. B 2020, 101, 174427 .

(63) Razdolski, I.; Alekhin, A.; Ilin, N.; Meyburg, J. P.; Roddatis, V.; Diesing, D.; Bovensiepen, U.; Melnikov, A. Nanoscale interface confinement of ultrafast spin transfer torque driving non-uniform spin dynamics. Nat. Commun. 2017, 8, 15007.

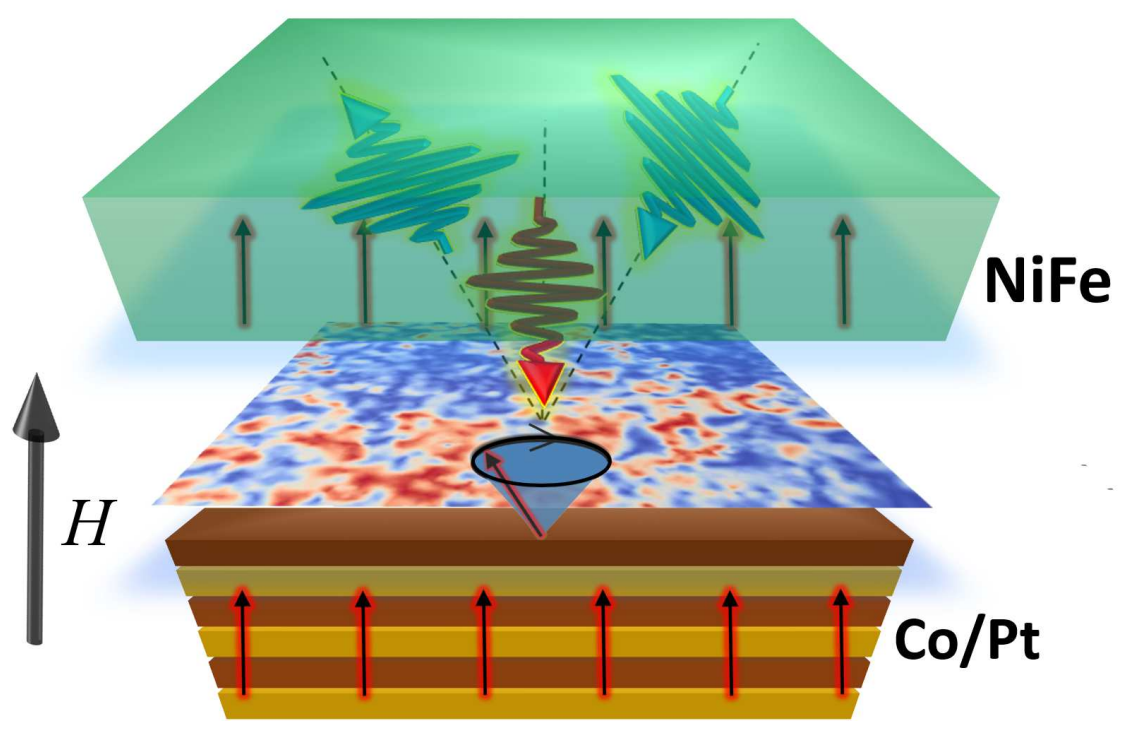

Figure 6: For Table of Contents Only 